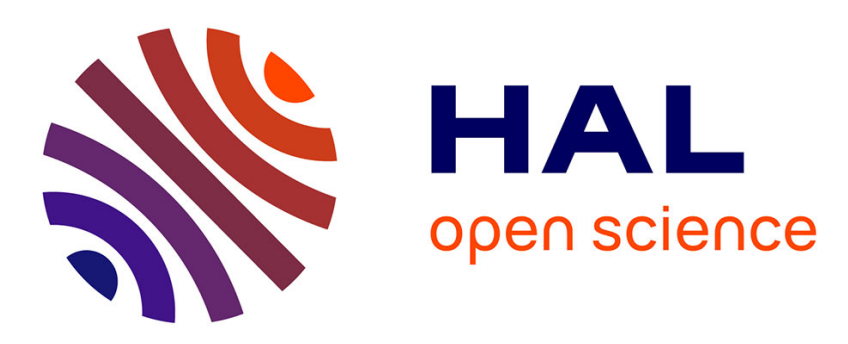

\title{
Les mobilisations de victimes de violences coloniales : investigations historiques et judiciaires et débats politiques postcoloniaux au Kenya
}

\author{
Marie-Emmanuelle Pommerolle
}

\section{- To cite this version:}

Marie-Emmanuelle Pommerolle. Les mobilisations de victimes de violences coloniales : investigations historiques et judiciaires et débats politiques postcoloniaux au Kenya. Raisons politiques, 2008, 30 (2), 10.3917/rai.030.0107 . hal-01648515

\section{HAL Id: hal-01648515 https://hal.science/hal-01648515}

Submitted on 26 Nov 2017

HAL is a multi-disciplinary open access archive for the deposit and dissemination of scientific research documents, whether they are published or not. The documents may come from teaching and research institutions in France or abroad, or from public or private research centers.
L'archive ouverte pluridisciplinaire HAL, est destinée au dépôt et à la diffusion de documents scientifiques de niveau recherche, publiés ou non, émanant des établissements d'enseignement et de recherche français ou étrangers, des laboratoires publics ou privés. 


\section{LES MOBILISATIONS DE VICTIMES DE VIOLENCES COLONIALES : INVESTIGATIONS HISTORIQUES ET JUDICIAIRES ET DÉBATS POLITIQUES POSTCOLONIAUX AU KENYA}

Marie-Emmanuelle Pommerolle

Presses de Sciences Po (P.F.N.S.P.) | « Raisons politiques »

2008/2 n³0 | pages 107 à 129

ISSN 1291-1941

ISBN 9782724631210

Article disponible en ligne à l'adresse :

http://www.cairn.info/revue-raisons-politiques-2008-2-page-107.htm

\section{Pour citer cet article :}

Marie-Emmanuelle Pommerolle, « Les mobilisations de victimes de violences coloniales : investigations historiques et judiciaires et débats politiques postcoloniaux au Kenya », Raisons politiques 2008/2 ( $\mathrm{n}^{\circ} 30$ ), p. 107-129. DOI 10.3917/rai.030.0107

Distribution électronique Cairn.info pour Presses de Sciences Po (P.F.N.S.P.).

(C) Presses de Sciences Po (P.F.N.S.P.). Tous droits réservés pour tous pays.

La reproduction ou représentation de cet article, notamment par photocopie, n'est autorisée que dans les limites des conditions générales d'utilisation du site ou, le cas échéant, des conditions générales de la licence souscrite par votre établissement. Toute autre reproduction ou représentation, en tout ou partie, sous quelque forme et de quelque manière que ce soit, est interdite sauf accord préalable et écrit de l'éditeur, en dehors des cas prévus par la législation en vigueur en France. Il est précisé que son stockage dans une base de données est également interdit. 


\section{MARIe-ÉmMANUelle Pommerolle}

\section{Les mobilisations de victimes de violences coloniales : investigations historiques et judiciaires et débats politiques postcoloniaux au Kenya}

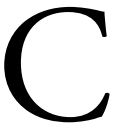

ETTE ÉTUDE S'ATTACHE À DÉCRIRE les processus de mobilisation autour d'ex-combattants mau mau insurgés contre la domination coloniale britannique au Kenya dans les années 1950. Ces mobilisations contribuent à faire de ces vétérans, comme ils se désignent eux-mêmes, les victimes d'une extrême violence déployée à leur encontre. Cette résurgence du passé colonial dans les débats politiques contemporains, au Kenya comme dans son ancienne métropole, nous renseigne sur deux éléments spécifiques aux mobilisations lancées au nom de victimes revendiquées. D'abord, la qualification même de victime est sujette à controverse et est apposée à partir de processus parfois contradictoires : ici, la recherche historique et l'action judiciaire sont mises à contribution pour affirmer le statut de victimes de ces vétérans mau mau, mais offrent des lectures contradictoires de cette victimisation. Ensuite, le statut de victime est l'objet d'usages multiples selon le lieu de son énonciation. Alors que des acteurs d'origines diverses (Kenyans, Britanniques, Américains) participent à ces mobilisations et que ces dernières se déploient au Kenya comme en Grande-Bretagne, les vétérans sont à la fois perçus 
comme des victimes d'une violence inouïe de la part de l'État colonial, et comme des héros dans le cadre national kenyan où leurs mobilisations actuelles ré-affirment le combat pour l'indépendance, l'un des éléments du récit de l'identité nationale. La construction ambivalente de cette figure de la victime autour des combattants mau mau est d'autant plus complexe que la nature de leur insurrection et des violences de cette époque sont toujours l'objet de débat, à la fois historique et politique.

Les Mau Mau, groupes armés présents à Nairobi et dans la province centrale majoritairement peuplée de Kikuyus, se sont attaqués à partir de 1952 à des colons et à des chefs locaux qui collaboraient avec la puissance coloniale. Les actes de répression perpétrés par l'administration coloniale et la mise en place d'une milice africaine loyaliste (les home guards) ont accentué les divisions au sein $\mathrm{du}$ groupe kikuyu. Les affrontements se sont poursuivis jusqu'en 1956 et les mesures répressives jusqu'en 1960. L'identification des victimes de ce conflit pose problème car d'un soulèvement de certaines catégories de la population Kikuyu (principalement) à l'encontre du pouvoir colonial, la violence s'est muée en un combat entre les Mau Mau et les individus qui avaient rapidement bénéficié de la colonisation. La nature et la qualification de ce conflit à la fois interne et anti-colonial ont fait débat pendant toute la période postcoloniale. En effet, alors qu'il a eu lieu avant l'indépendance, ses conséquences ont joué un rôle déterminant lors de la redistribution du pouvoir après le départ des Britanniques en 1963. Ce sont alors les nationalistes très modérés - parmi lesquels le Président Jomo Kenyatta - qui ont pris le pouvoir, tandis que certains loyalistes conservaient un pouvoir économique, notamment foncier, et ont pu bénéficier des faveurs du premier gouvernement postcolonial. Si les deux premiers présidents, Kenyatta (1964-1978) et Moi (1978-2002), se sont référés ponctuellement à l'insurrection mau mau pour légitimer leur pouvoir, ils ont préféré mettre de côté cet épisode conflictuel de l'histoire kenyane ; ce sont avant tout les opposants qui, se proclamant héritiers des freedom fighters, ont utilisé leurs symboles et leurs héros pour stigmatiser l'autoritarisme postcolonial ${ }^{1}$.

1. Voir Marshall S. Clough, "Mau Mau and the Contest for Memory ", in John Lonsdale et Atieno E. S. Odhiambo (dir.), Mau Mau and Nationhood: Arms, Authority and Narration, Londres, James Currey, 2003, p. 251-267 et Mau Mau Memoirs : History, 
L'objectif de cette réflexion sera à la fois de comprendre les ressorts variés de la qualification de ces ex-combattants mau mau comme victimes de la violence coloniale, et les conditions des mobilisations autour de ces victimes. Nous verrons en premier lieu que deux logiques, l'une historique et l'autre judiciaire, s'affrontent pour participer à la désignation des victimes et aboutissent à des " vérités " contradictoires. L'implication de personnalités étrangères (des «experts" britanniques et américains, spécialistes du Kenya) accentue les lectures divergentes de ces mobilisations et introduit des débats sur la légitimité de ceux qui parlent au nom de ces "victimes ». Ce surgissement du passé dans les débats scientifiques mais aussi publics (prises de parole de membres du gouvernement, articles dans les presses nationales kenyane et britannique, événements organisés par les ONG qui s'occupent de ces mobilisations) est, comme nous le verrons en second lieu, le fruit de configurations politiques contemporaines. Ces mobilisations s'accentuent en effet depuis 2003 et l'alternance politique qu'a connue le Kenya après la défaite de l'ancien parti unique ${ }^{2}$. Hervé Maupeu propose de voir dans ce retour du passé une sorte de "catharsis " post-alternance : dénoncer les méfaits de cette période revient à dénoncer les erreurs et les injustices des régimes postcoloniaux tant les deux périodes sont liées, dans les faits et dans les représentations politiques kenyanes $^{3}$. La transition a ainsi constitué une opportunité politique pour nombre d'acteurs qui voyaient dans l'ancien régime la continuation de la situation coloniale; s'emparer du passé est aussi un moyen de renouveler des revendications dans un contexte où l'ennemi n'est plus le même, ou encore de trouver un registre de

Memory and Politics, Londres, Lynne Rienner publishers, 1998 ; Galia Sabar-Friedman, "The Mau Mau Myth ", Cahier d'études africaines, no 137, 1995, p. 101-131.

2. Une coalition d'opposition, la National Rainbow Coalition (Narc), a gagné les élections de décembre 2002 contre l'ancien parti unique, la Kenya African National Union (Kanu). Le candidat de la Narc, Mwai Kibaki, un Kikuyu, est devenu président de la République, remplaçant Daniel Arap Moi, un Kalenjin, qui occupait cette fonction depuis 1978. Le retour d'un président kikuyu au pouvoir permettra aux revendications, portées par des Kikuyus, ethnie largement majoritaire chez les Mau Mau, de se faire mieux entendre. À propos de cette alternance, voir notamment Hervé Maupeu et David Anderson, "Kenya : la succession de Moi ", Politique africaine, $\mathrm{n}^{\circ}$ 90, juin 2003, p 5-16 ; Stephen N. Ndegwa, "Kenya : Third Time Lucky? ", Journal of Democracy, vol. 14, no 3, 2003, p. 145-158; Stephen Brown, "Theorising Kenya's Protracted Transition to Democracy", Journal of Contemporary African Studies, vol. 22, $\mathrm{n}^{\circ} 3$, 2004, p. 325-342.

3. Hervé Maupeu, "Le régime Kibaki - An I", Annuaire de l'Afrique orientale 2003, Paris, L'Harmattan ; Pau, CREPAO ; Nairobi, IFRA, 2004, p. 161-188. 
légitimation apparemment inoffensif pour le nouveau gouvernement. Ce débat sur les victimes, qui rejoint celui sur les héros nationaux, prolonge également un questionnement sur l'héritage contemporain de la période coloniale et soulève donc des questions politiques cruciales, montrant ainsi que les mobilisations de victimes ne sont pas apolitiques.

\section{Désignation et fabrication des victimes : les controverses historiques}

Les historiens kenyans et étrangers ont proposé des interprétations variées sur l'insurrection mau mau. Les historiens marxistes ont décrété que ce soulèvement s'apparentait à une lutte de classes, d'autres y ont vu un véritable moment nationaliste ; d'autres encore, jugés " conservateurs " par les premiers, ont démontré qu'il ne s'agissait là que d'un conflit interne au groupe kikuyu qui ne concernait pas l'ensemble de la population kenyane ${ }^{4}$. Des ouvrages plus récents tentent de rassembler ces approches et de les faire communiquer ${ }^{5}$. Deux ouvrages publiés en 2005 ont réactivé les débats scientifiques et médiatiques au sujet de l'insurrection des années 1950 en levant le voile sur un thème peu étudié jusqu'ici : la répression de l'insurrection. Britain's Gulag ${ }^{6}$ de Caroline Elkins et Histories of the Hanged $^{7}$ de David Anderson s'attaquent tous deux à l'interprétation de ces affrontements diffusée dans les années 1950 en GrandeBretagne, qui faisait des colons les principales victimes de cette violence. Ils insistent sur la responsabilité des colons et de l'administration coloniale dans ces violences, alors que les Britanniques envisageaient à l'époque le soulèvement mau mau comme le symptôme d'une maladie psychosociale, conséquence d'une confrontation trop rapide à la modernité, et ne remettaient pas en cause le bien-fondé de leur présence au Kenya. Les deux ouvrages

4. E. A. S. Odhiambo, "The Production of History in Kenya : the Mau Mau Debate", Canadian Journal of African Studies, vol. 25, $\mathrm{n}^{\circ}$ 2, 1991, p. 300-307 ; Gérard Prunier, "Mythe et histoire : les interprétations du mouvement Mau Mau de 1952 à 1986 ", Revue française d'histoire d'outre-mer, vol. 74, n' 277, 1987, p. 401-429.

5. Voir J. Lonsdale et E.A.S. Odhiambo (dir.), Mau Mau and Nationhood..., op.cit.

6. Caroline Elkins, Britain's Gulag: The Brutal End of Empire in Kenya, Londres, Jonathan Cape, 2005.

7. David Anderson, Histories of the Hanged. The Dirty War in Kenya and the End of Empire, New York, Londres, Norton \& Co, 2005. 
s'accordent sur les responsabilités britanniques et sur le fait que les victimes se trouvaient avant tout chez les Africains.

Mais les deux auteurs construisent ensuite des hypothèses divergentes sur la définition précise des victimes. Anderson travaille à partir de "témoignages des deux camps " et assure dès le début de son ouvrage que "c'est une histoire d'atrocités des deux côtés, une sale guerre de laquelle personne ne sort avec fierté, ou avec gloire. C'est une histoire gênante, mais qui doit être racontée » (p. 2). De son côté, Elkins veut revenir sur un passé caché volontairement par les Britanniques, celui de leur volonté d'anéantissement des Kikuyus, à travers les camps de détention et les villages d'urgence. Elle cherche à démontrer que les Kikuyus, Mau Mau et leurs familles, étaient les cibles d'une répression masquée derrière un discours de "réhabilitation ". Elle montre que si les colons considéraient les Kikuyus comme une nuisance à éliminer (p. 49), l'administration coloniale ne dessinait pas de tels projets mais finit, en pratique, par y souscrire. L'auteur reprend en fait la catégorie définie par les bourreaux britanniques: tous ceux qui sont morts, ont été enfermés ou ont subi les agressions physiques et morales (destruction des biens, déplacements forcés) de la part de l'administration britannique et de ses alliés sont des victimes, à reconnaître comme telles. De plus, elle évacue d'emblée la question de la violence perpétrée par les Mau Mau (p. xiv) en assumant de suspendre son regard critique et en entourant les Mau Mau d'un " halo d'innocence " propice à leur représentation en tant que victimes ${ }^{8}$. Anderson, lui, remet en cause ces catégories à partir d'une relecture historique et sociale de certains jugements prononcés durant la répression. Le statut de victimes, dans son ouvrage, n'est pas posé mais remis en cause. Il montre par exemple que ceux qui ont été victimes de la justice de la colonie britannique ont également pu être victimes des Mau Mau ou que ceux qui ont trahi ces Mau Mau ont aussi pu être victimes de l'appareil policier britannique ; il soutient en définitive que l'ensemble de la population a été victime d'une situation historique violente, de la colonisation et de ses dérives.

Un débat historiographique et médiatique s'est engagé dans les recensions comparant les deux ouvrages et critiquant, le plus souvent, l'ouvrage d'Elkins. Si ce sont souvent les méthodes qui

8. Voir Philippe Braud, Violences politiques, Paris, Seuil, 2004, p. 208. 
sont attaquées, il semble que ce soit en fait la démonstration d'Elkins sur la violence volontairement déployée par l'État britannique qui déplaise, surtout dans les journaux britanniques ${ }^{9}$. Une comparaison rapide de ces méthodes permet de mieux comprendre comment chacun construit sa conception des "victimes". L'argumentaire historique de chacun des auteurs les conduit à se fonder sur des sources différentes, à produire des bilans chiffrés de la répression divergents, et à s'appuyer sur des comparaisons historiques controversées. Le premier débat méthodologique porte sur la nature des sources historiques utilisées. L'ouvrage d'Anderson repose principalement sur les archives judiciaires de procès intentés à des Mau Mau présumés, qui ont été jugés coupables et pendus. Peu de recensions font mention de l'éventuelle partialité de ces sources coloniales que l'historien recoupe et enrichit afin de remettre en cause les sentences. L'ouvrage d'Elkins part justement de l'absence d'archives crédibles sur la répression (elles auraient été éliminées ou seraient partiales, p. 372) et cherche à combler le défaut de sources écrites par des témoignages oraux qui constituent l'originalité de son travail. Ces derniers sont fondamentaux dans sa démonstration : récits de torture, d'enfermement, de vie quotidienne au sein des camps et des villages de détention. Cette " histoire du sensible ", analysée par Hélène Charton, participe à la fabrication de ce statut de victimes conféré à tous ceux qui ont été enfermés par l'administration britannique ${ }^{10}$. Le second débat méthodologique porte sur le calcul du nombre de victimes de la violence coloniale sur lesquels les deux auteurs reviennent. Tout en soulignant qu'elle n'a pas les moyens scientifiques d'avancer des chiffres précis, Elkins conteste plusieurs bilans antérieurs. Mais ses modes de calcul sont contestés ${ }^{11}$. La réponse de John Nottingham, ancien district officer au Kenya à cette époque, qui soutient le travail de Caroline Elkins, révèle les enjeux de ces batailles de chiffres. Pour lui, s'ils ne sont pas exacts, les chiffres d'Elkins sont plus proches de la vérité que ceux du rapport

9. Voir les recensions critiques, principalement dans des journaux britanniques, "How not to run an empire ", The Economist, 29 décembre 2004 ; "A very British Gulag ", Times on Line, 8 janvier 2005; The Sunday Times, 9 janvier 2005 ; " Forgotten shame of Empire", The Independent, 21 janvier 2005 ; "State of Shame", The Guardian, 5 février 2005; London Review of books, 26 février 2005; et les avis partagés dans "The Dark Side of the Empire", Daily Telegraph, 11 janvier 2005 et "They Died Cursing the British ", Daily Telegraph, 16 janvier 2005.

10. Hélène Charton, "Les Mau Mau entre histoire et mémoire ", à paraître.

11. Voir les articles critiques sur l'ouvrage publiés dans le New York Review of Books, 7 avril 2005 et 23 juin 2005. 
Corfield, le rapport officiel de l'administration britannique sur l'insurrection et la répression ${ }^{12}$. Ainsi, l'exactitude des faits historiques n'apparaît pas primordiale, du moment qu'ils servent une démonstration ${ }^{13}$. Le dernier débat autour de Britain's Gulag est conceptuel : il porte sur les rapprochements que l'auteur effectue, ponctuellement, entre la violence subie par les Kikuyus et celle vécue par les Arméniens ou les Juifs, victimes de génocides. Ce procédé, plus rhétorique que scientifique, tend à souligner le caractère volontaire de la violence des Britanniques à l'encontre d'une population ciblée, mais il flirte parfois avec la légèreté théorique pour mieux servir son propos. Les différences méthodologiques portées par deux projets historiographiques n'ont pas échappé aux recensions très critiques envers l'ouvrage de Caroline Elkins. Elles remettent en cause l'" objectivité » de son travail : "Elkins n'a pas le temps de s'attarder sur les atrocités commises par les Mau Mau; de même, elle n'a pas de temps pour les loyalistes qui ont eu peur des Mau Mau ${ }^{14}$. "À l'inverse de Histories of the Hanged de David Anderson qui serait " équilibré " car il reconnaît la violence des deux côtés, on reproche à Elkins d'avoir produit un essai " unidimensionnel ${ }^{15}$ " dû à sa " colère $^{16}$ ". Ce mode de discours révolté sur la violence passée, s'il est mitigé par une démarche scientifique, participe à la construction de ce statut de victime qu'Anderson, pour sa part, refuse de conférer à un groupe particulier.

\section{Histoire et justice : des vérités contradictoires sur les victimes}

Les vérités historiques sur la désignation des victimes de cette violence coloniale apparaissent d'autant plus contradictoires qu'elles se construisent en référence à des vérités judiciaires qu'elles entendent critiquer dans le cas de David Anderson, qui revient sur les procédures judiciaires passées, et servir dans le cas d'Elkins, qui met à disposition ses recherches dans le cadre d'une plainte déposée à l'encontre de l'État britannique.

12. Voir London Review of Books, 7 juillet 2005.

13. Ph. Braud, Violences politiques, op.cit, p. 22.

14. Daily Nation, 31 janvier, 2005.

15. The Guardian, 5 février 2005.

16. Daily Nation, 31 janvier, 2005. 


\section{L'histoire de la justice ou l'impossibilité de statuer sur les catégories de victimes et de bourreaux}

David Anderson se base sur les archives des procès d'environ $1000 \mathrm{Mau}$ Mau qui ont été exécutés, et revisite le procès d'un ensemble de condamnés. Sa démonstration remet en cause certains jugements, par l'exposition de nouvelles données, et apporte une perspective d'histoire sociale, et non individuelle, sur les responsabilités et la "culpabilité " de chacun ${ }^{17}$. Son chapitre central, consacré à un double massacre perpétué par des Mau Mau et par des loyalistes, met particulièrement bien en lumière l'apport de l'historien face aux vérités judiciaires passées. L'attaque de combattants mau mau sur le village de Lari, en mars 1953, laissant 120 morts dont une majorité de femmes et d'enfants, puis la vengeance meurtrière à l'égard de ceux que les home guards tenaient pour responsables, ne peuvent être lues au regard des condamnations des supposés responsables mau mau, dont Anderson scrute les procès, mais doivent être replacées dans leur contexte historique qui, sans pouvoir déterminer précisément les responsabilités individuelles, permet de nuancer les sentences injustes et partiales de la justice coloniale. L'historien émet des hypothèses sur les causes de cette violence particulière, en relatant l'histoire de cette communauté profondément divisée socialement, politiquement et religieusement. Il explique la cruauté de l'attaque mau mau par le fait qu'elle visait les chefs de clan, hommes d'Église, propriétaires fonciers, symboles de leur propre dépossession, et suggère que le meurtre de leurs femmes et de leurs enfants correspondait à une volonté d'interrompre la transmission des biens fonciers dans les familles loyalistes. Une deeper history qui remet en cause les motifs évoqués lors des procès des accusés. Surtout, en examinant les comptes-rendus des procès des 309 Mau Mau accusés d'être impliqués dans le massacre, il s'interroge sur la culpabilité des pendus qui, pour beaucoup, n'ont été accusés que sur la base de témoignages de survivants qui les connaissaient intimement et qui ont été profondément affectés par le massacre. Ces villageois auraient donc payé pour les véritables groupes armés mau mau qui avaient déserté la scène de leurs crimes, laissant les villageois à la merci des home guards, puis de la justice (p. 178). C'est ainsi qu'aux procès, les accusés « ne sont pas

17. Voir Jean-Clément Martin, «La démarche historique face à la vérité judiciaire. Juges et historiens ", Droit et Société, no 38, 1998, p. 13-20. 
conformes à l'image habituelle de l'héroïque freedom fighter" (p. 175) : si certains étaient des Mau Mau convaincus, d'autres étaient plus réticents et avaient participé sans conviction au massacre. Pourtant, "les comptes-rendus des procès de Lari ne nous permettent pas de faire aisément la distinction entre ces catégories de gens » (p. 175); ce n'est qu'en s’interrogeant à partir des témoignages contradictoires et du contexte socio-historique de ces massacres que la qualité de victimes et de bourreaux peut être affinée, sans qu'aucune vérité, ni judiciaire, ni historique, ne puisse être définitivement arrêtée. "Les accusés sont-ils coupables?" se demande David Anderson, avant de conclure que demeurent de nombreux secrets, que ni la justice, ni l'histoire ajouterons-nous, n'ont pu et ne pourront dévoiler (p. 176).

\section{Le recours à la justice contemporaine : des victimes visibles}

La publication de l'ouvrage d'Elkins constitue le volet académique d'un engagement plus large pour la reconnaissance des crimes britanniques commis au Kenya. C'est en collectant des témoignages pour sa thèse de doctorat que l'historienne a décidé d'en faire également un combat militant. Elle a par exemple participé à la production d'un documentaire diffusé sur la BBC en novembre 2002 décrivant la répression britannique envers les Mau Mau. Ce documentaire avait conduit Scotland Yard à mener une première enquête, restée sans suite, sur les crimes commis durant la répression britannique ${ }^{18}$. La médiatisation des recherches d'Elkins, notamment depuis l'attribution du prix Pulitzer à son ouvrage en 2006, est un atout majeur pour les juristes qui ont multiplié les actions auprès de la justice britannique, notamment à partir des témoignages collectés par l'historienne.

Appuyée sur quelques cas avérés de torture commis dans des camps de détention durant l'insurrection mau mau, cette plainte s'appuie sur le droit international et la common law pour accuser l'État britannique de négligence et de complicité d'actes de torture. Conformément à la vision qu'en donne Caroline Elkins dans son ouvrage, l'État britannique est accusé d'avoir été un État

18. Le documentaire a reçu en 2003 le prix de la Croix rouge internationale au festival de Monte-Carlo. 
tortionnaire et les Mau Mau ne sont logiquement désignés, dans ce procès, que comme les victimes de cet État bourreau. Cette présentation est considérée comme insuffisante par David Anderson qui, interrogé par la BBC lors du dépôt au Foreign Office de la "Letter of claims ${ }^{19}$ " par l'avocat des vétérans mau mau, insiste sur les souffrances connues par les deux camps lors de l'insurrection : "Il y a eu beaucoup de souffrance des deux côtés. C'était une sale guerre. C'est devenu un guerre civile. Mais cette idée est très impopulaire au Kenya aujourd'hui ${ }^{20}$." Ce à quoi l'avocat britannique des Mau Mau répond, dans le même article de presse : "La part d'atrocités commises par les Mau Mau est infime comparée à celle des Britanniques (...). Pour les premières, on parle de dizaines de gens, pour les secondes, de dizaines de milliers. " La "Letter of claims " ayant reçu une fin de non recevoir de la part du Foreign Office en avril $2007^{21}$, les avocats devaient déposer une plainte en justice au premier semestre $2008^{22}$. On le voit, cette affaire permet de conforter le découpage effectué par l'historienne entre, d'un côté, les victimes que sont les Mau Mau et, de l'autre, le reste de la population et l'État, considérés comme de potentiels coupables.

Le choix sélectif des plaignants et des témoins est venu encore renforcer cette victimisation des vétérans mau mau, réduits à n'être que les traces de la violence déployée sur leur corps et sur leur groupe. Le choix a été fait de se concentrer sur les cas de torture, car cette dernière laisse des marques visibles, seules preuves tangibles, alors que les actes incriminés ont été commis il y a cinquante ans. Cette sélection des victimes pour le procès n'est pas sans produire des justifications gênées de la part de ses initiateurs, obligés de mettre de côté les personnes décédées et les pertes de biens,

19. Une "Letter of claims " a été déposée au ministère des Affaires étrangères (Foreign Office) le 12 octobre 2006 afin d'exposer l'intention des plaignants d'attaquer l'État britannique en justice pour actes de torture et de demander à ce dernier de reconnaître ses torts. Cette étape est purement formelle.

20. "Bloody Uprising of the Mau Maus », 12 octobre 2006, collecté sur www.bbc.co.uk en octobre 2006 (notre traduction).

21. La réponse à la «Letter of claims " datée du 2 avril 2007 met en avant l'impossibilité pour le Foreign Office de présenter des preuves contradictoires face aux éléments présentés par les plaignants (les traces de torture) ainsi que la prescriptibilité des crimes mis en cause.

22. La justice britannique sera alors susceptible d'opposer deux objections à cette plainte : le transfert de souveraineté (l'État britannique de l'époque est devenu l'État kenyan) et la prescriptibilité des crimes. 
notamment d'animaux et de terres, subies par les anciens combattants mau mau et leur familles ${ }^{23}$. Seuls ceux qui sont capables d'exhiber les signes de la violence subie sont considérés comme des victimes utiles. Douze vétérans seront amenés en tant que plaignants en Grande Bretagne lors de l'exposé des motifs de la plainte : le choix a été fait de se concentrer sur quelques cas, à propos desquels les juristes n'ont aucun doute et assez d'éléments pour avérer les faits de torture. Cette sélection des victimes utiles et efficaces s'est faite à partir de quelques témoignages présents dans l'ouvrage de Caroline Elkins, complétés par de longues séances d'entretiens afin de s'assurer de la crédibilité des témoignages.

À l'image des vétérans des colonies françaises ayant combattu durant la Seconde Guerre mondiale et montrés au public comme preuves vieillissantes d'une injustice ${ }^{24}$, ces vétérans mau mau témoignent, mais surtout incarnent ce combat et l'injustice dont ils auraient été victimes. Présentés à plusieurs reprises lors du Forum social mondial qui se tenait à Nairobi en janvier 2007, les vétérans mau mau sont apparus avant tout comme les dépositaires physiques d'un combat passé. Lors d'un atelier sur les "mémoires des combats ", le public a écouté les courts témoignages des vétérans mau mau et s'est enthousiasmé de leur présence physique et de leur image : ils ont été pris en photo pendant plusieurs minutes, les flashs redoublant lorsque l'une des femmes mau mau ôta son foulard et montra ses dreadlocks, symboles de son appartenance au mouvement. Vieillis, habillés pauvrement, mais toujours prêts à montrer les signes corporels de leur engagement et à exhiber leur fierté intacte (à travers des chants et des prières), ces vétérans figurent à la fois l'héroïsme et la pitié, celle due à la victime d'une injustice. Au-delà de leurs apparitions, organisées par ceux qui estiment avoir poursuivi leur combat ${ }^{25}$, les vétérans ne sont plus au

23. Entretien avec le responsable de ce procès à la Kenya Human Rights Commission, Nairobi, 12 janvier 2007.

24. Je fais référence ici aux apparitions médiatiques des vétérans lors de la sortie du film Indigènes et de la décision politique d'augmenter la pension de ces anciens combattants.

25. Lors du Forum social mondial, ce sont en effet d'anciens prisonniers politiques, aujourd'hui militants d'organisations des droits de l'Homme, qui se sont chargés des déplacements des vétérans Mau Mau, qui habitent tous dans la région centrale. Ces éléments sur les Mau Mau au Forum social mondial ont été collectés lors d'une enquête de terrain effectuée dans le cadre du projet de recherche "Causes africaines : mobilisations, courtage international et luttes sur les formes légitimes de gouvernement en Afrique» financé par l'Agence nationale de la recherche, en janvier 2007. 
cœur de ces actions de reconnaissance, mais ils en sont la justification historique et physique. Ce sont avant tout des acteurs militants, juristes, associatifs, ou témoins des violences passées qui se sont saisis d'une nouvelle opportunité politique, l'alternance de 2002, pour faire entendre des revendications, déjà formulées mais peu entendues auparavant.

\section{Temporalités et stratégies politiques autour des mobilisations de victimes}

L'alternance de décembre 2002, qui a vu la défaite de l'ancien parti unique et l'arrivée au pouvoir d'une coalition d'opposition, a provoqué et encouragé au Kenya l'émergence d'un ensemble de mobilisations. Alors que les régimes de Kenyatta et Moi étaient perçus par leurs opposants comme les prolongements directs de l'autoritarisme colonial, l'alternance a pu, au début, faire croire à l'avènement d'un État démocratique remplaçant l'"État bourreau ». Les victimes ou leur porte-parole se sont adressé à ce nouvel arbitre, qui a joué sur cette nouvelle image, tout en se gardant bien de répondre positivement aux requêtes foncièrement politiques de ces victimes passées. Or, une fois engagées, ces mobilisations de victimes de la violence coloniale réactivent des débats autour du passé et de la légitimité des gouvernants actuels. La mobilisation autour de victimes n'est pas apolitique, bien au contraire.

\section{Des mobilisations de transition}

Le gouvernement kenyan d'alternance a très rapidement cherché à obtenir un supplément de légitimité à travers le soutien apporté aux revendications des victimes de violences coloniales et postcoloniales. La volonté du nouveau gouvernement de rendre justice aux victimes de crimes passés s'est manifestée par l'ouverture d'anciens lieux de détention et de torture utilisés par le régime moi $^{26}$, et par la mise en place d'une commission chargée de réfléchir à la nécessité d'établir une Commission Vérité, Justice et

26. «Kenya Torture Cells to Become Monument », Sunday Times, 16 février 2003. 
Réconciliation. Malgré un avis positif ${ }^{27}$, le gouvernement n'a cependant pas jugé nécessaire de suivre ces recommandations. Le caractère hétéroclite de la coalition victorieuse en 2002, où cohabitaient anciens opposants et membres des régimes antérieurs, a semblé atténuer la volonté d'investigation des crimes perpétrés sous les régimes postcoloniaux $^{28}$. La transformation de l'« État bourreau " en État démocratique s'est avérée en fait toute relative : la permanence d'une partie du personnel politique, anciens membres de la Kenya African National Union, a empêché certaines investigations et les enjeux de fond portés par ces revendications n'ont pu être assumés par l'élite politique au pouvoir. Le Président a bien encouragé, très tôt, les initiatives symboliques de reconnaissance des freedom fighters: la recherche (vaine) du corps de Dedan Kimathi, un leader mau mau pendu par les Britanniques, le changement du "Kenyatta Day» en "Majusha Day» ("Jour des Héros»), la reconnaissance juridique des associations de vétérans, sur lesquelles nous revenons ensuite, ont semblé exprimer une ouverture nouvelle du gouvernement au débat sur la mémoire mau mau, qui répondait aux demandes de groupes de militants ayant largement soutenu la coalition victorieuse. Mais ce geste d'ouverture n'a pas résisté aux intérêts stratégiques et politiques d'un gouvernement fragilisé par les scandales de corruption et par de vives oppositions.

Lors du lancement de l'ouvrage de Caroline Elkins en mars 2005 à Nairobi, le ministre de la Justice et le vice-président ont certes demandé publiquement au gouvernement britannique de s'excuser pour les crimes commis envers les Mau Mau ${ }^{29}$. Les oppositions internes au sein de la coalition gouvernementale ont même semblé pouvoir être temporairement camouflées par ces annonces aux accents anti-coloniaux plutôt consensuels, dans un contexte tendu entre le gouvernement et l'ambassadeur britannique. Ce dernier avait en effet accusé en juillet 2004 et en février 2005

27. Republic of Kenya, Report of the Task Force on the establishment of a Truth, Justice and Reconciliation commission, Nairobi, 2003.

28. On trouve aussi bien des anciens ministres de la KANU, passés à l'opposition quelques mois avant les élections de 2002, que des opposants plus anciens et plus déterminés. De plus, les changements d'alliance depuis 2002 ont favorisé le retour d'anciens membres de la KANU au sein du gouvernement. Voir, par exemple, Godwin R. Murunga et Shadrack W. Nasong'o, "Bent on Self-Destruction : The Kibaki Regime in Kenya ", Journal of Contemporary African Studies, vol. 24, n 1, janvier 2006, p. 1-28.

29. "Kenya Wants UK “Atrocity" Apology ", BBC News, 4 mars 2005. 
les membres du gouvernement d'être corrompus, en des termes jugés "grossiers" par de nombreux observateurs kenyans ${ }^{30}$. La démission du responsable de la lutte anti-corruption au sein du gouvernement kenyan, John Githongo, et son " exil » volontaire en Grande-Bretagne soulignèrent les tensions existant entre les deux pays à propos de la corruption, l'un des thèmes majeurs de la "bonne gouvernance " imposée par les bailleurs de fonds au Kenya, comme à de nombreux autres pays bénéficiaires de l'aide internationale. Ces frictions se retrouvèrent dans la réaction de l'un des porte-parole du Foreign and Commonwealth Office qui, interrogé à propos des discours véhéments des officiels kenyans à propos des violences coloniales, affirma sur $B B C$ News que le gouvernement britannique comprenait la souffrance invoquée par les victimes, tout en appelant à l'oublier : "Ceci s'est passé il y a cinquante ans. Le Kenya doit maintenant se préoccuper des défis futurs comme la lutte contre la corruption, l'injustice et la construction d'une démocratie stable ${ }^{31}$. " Loin d'endosser le costume de responsable, le gouvernement britannique semble ainsi rejeter les interpellations. Le gouvernement kenyan n'est cependant pas prêt à se brouiller avec l'ancienne métropole à propos de ces enjeux passés, alors que les soutiens diplomatiques et financiers sont bien plus importants pour la survie politique des dirigeants actuels, notamment parce que les entreprises britanniques sont les premiers investisseurs étrangers dans le pays. Néanmoins, si les prises de position officielles ne montrent pas de soutien direct à la plainte déposée contre l'État britannique, des membres du gouvernement ont soutenu personnellement cette initiative lors d'un dîner de levée de fonds organisé par la Kenya Human Rights Commission (KHRC), l'organisation de défense des droits de l'Homme kenyane qui organise l'action judiciaire $^{32}$.

30. Les propos du Haut Commissaire tenus en juillet 2004 peuvent être traduits de la sorte : "La corruption massive au Kenya revient à vomir sur les chaussures des bailleurs, sur celles de tous les Kenyans, et sur les pieds de ceux qui n'ont pas les moyens de se payer des chaussures... ». Voir www.bbc.co.uk, archives de juillet 2004 consultées en mars 2006; The East African, 28 février 2005.

31. "Kenya wants UK "atrocity" apology ", BBC News, 4 mars 2005.

32. Un million de Kenyan Shillings (Ksh) a été collecté lors de ce dîner ; le coût estimé de cette action en justice serait d'environ 17 millions Ksh (180 000 euros), et, en janvier 2007, il manquait 11 millions Ksh aux initiateurs du procès; l'agence de coopération danoise DANIDA finance l'aspect logistique de ce projet à hauteur de 5 millions de Ksh mais ne veut pas que ses fonds soient utilisés pour payer l'avocat britannique, dont les honoraires s'élèvent à 6 millions de Ksh. Certains hommes 
Ce soutien discret déçoit les porte-parole de ces mobilisations, notamment les organisations de défense des droits de l'homme qui ont toujours défendu la reconnaissance des Mau Mau, dont elles se disent héritières, et qui ont soutenu en 2002 la coalition victorieuse. Depuis le milieu des années 1990, diverses organisations de défense des droits de l'homme, ONG soutenues par des bailleurs internationaux et groupes de jeunes radicaux, ont exigé auprès des Britanniques et de l'État kenyan que soit retrouvé le corps de Dedan Kimathi, afin qu'il bénéficie de funérailles dignes de son combat. Ce leader Mau Mau a été élevé au rang de héros de l'insurrection au fil du temps, notamment à travers les écrits de l'écrivain kenyan Ngugi wa Thiong'o, qui en a fait le martyre d'une cause nationaliste et populaire ${ }^{33}$. Présentes dans les écrits des ONG de défense des droits de l'homme, comme la KHRC ou Release Political Prisoners qui ont toutes deux été à la pointe des revendications démocratiques dans les années 1990, les références aux Mau Mau viennent ancrer leurs revendications dans une généalogie historique et héroïque de l'opposition kenyane. Pour la KHRC, parler de Dedan Kimathi revient à parler des droits de l'homme : il représenterait en effet à la fois la lutte contre la peine de mort (il a été pendu par les Britanniques) et l'opposition à l'accaparement foncier mené par les colons et ses prolongements contemporains ${ }^{34}$. On le verra, ce recours à des figures sélectionnées et héroïsées n'est pas sans poser problème ; néanmoins, cette filiation historique et son usage symbolique ont permis aux ONG de défense des droits de l'homme de gagner une audience nationale face aux critiques présentant les droits de l'homme comme une cause importée.

Dans la lutte de la KHRC pour la défense des droits de l'homme, et plus précisément contre les exactions menées par le régime du président Moi, la collecte d'informations en vue du procès contre l'État britannique constitue donc la suite de ces réactivations de la mémoire mau mau. À mesure qu'approchait l'éventuelle l'alternance, la KHRC s'était intéressée à la question de l'impunité des

politiques kikuyu auraient proposé de financer le procès, mais cette proposition a été refusée par ses initiateurs, de peur de devenir otages de stratégies politiciennes.

33. Voir par exemple, Ngugi wa Thiong'o et Mugo Micere, The Trial of Dedan Kimathi, Nairobi, East African Educational Publishers, 1976.

34. Willy Mutunga et Alamin Mazrui, "Rights Integration in an Institutional Context ", non publié, 1999 collecté à la KHRC, organisation dont les auteurs sont membres fondateurs. 
crimes commis par le régime moi $^{35}$, qui risquaient de ne pas être sanctionnés après le départ du Président. À côté d'une campagne lancée en 2001 sur "l'éradication de la culture de l'impunité " et d'un travail de recherche et de lobbying pour la mise en place d'une commission Vérité, Justice et Réconciliation enquêtant sur les crimes postcoloniaux, cet intérêt des ONG de défense des droits de l'Homme kenyanes pour les crimes coloniaux est une autre manière d'exprimer leur refus de l'un des slogans du candidat Kibaki, victorieux Président, "forgive and forget» ("Pardonne et oublie»), un slogan se rapportant aux crimes commis par le régime moi, mais qui réactivait la formule prononcée par Jomo Kenyatta au moment de l'indépendance ${ }^{36}$. Alors que les violences britanniques et les conflits internes aux sociétés de la province centrale ont été écartés du débat postcolonial, le candidat de la coalition d'opposition, quarante ans plus tard, proposait également de mettre de côté les exactions et les conflits du régime qu'il combattait. Cette position a été comprise comme un moyen d'apaiser cette période transitionnelle en assurant l'ancien Président, qui n'avait plus le droit de se présenter aux élections, d'un avenir paisible. Les militants des droits de l'homme, qui ont soutenu la coalition d'opposition, ont refusé cette impunité déclarée. Après d'intenses débats, le président de la KHRC, Makau wa Mutua, professeur de droit à la State University de New York, a été nommé en 2003 président d'une commission chargée de travailler sur la nécessité et sur les modalités d'établissement d'une commission Vérité, Justice et Réconciliation. La participation de ces mêmes militants au procès des Mau Mau peut être vue comme une déclinaison sur le plan historique de ce refus catégorique de l'impunité. C'est également un moyen de redéfinir les objectifs et les activités de ces groupes, au moment où ils ont perdu leur principal ennemi, le régime moi. Cohérence des revendications et déplacement stratégique peuvent ainsi expliquer cette nouvelle orientation des militants des droits de l'homme kenyans.

Principalement dirigées par des juristes, universitaires et praticiens, les ONG de défense des droits de l'homme kenyanes ont

35. Ces organisations s'intéressent à la responsabilité de personnalités politiques aussi bien dans les conflits ethno-politiques des années 1990 que dans la corruption ou l'accaparement foncier, par exemple.

36. "Why a Truth Commission Will Help Break with the Past", Daily Nation, 2 décembre 2002. 
toujours travaillé en collaboration avec des cabinets d'avocats (celui de Gibson Kamau Kuria ou de Paul Muite) et se sont engagées dans plusieurs batailles juridiques (notamment la revendication constitutionnelle) ${ }^{37}$. On retrouve donc parmi les "têtes pensantes" de cette action judiciaire, Willy Mutunga et Maina Kiai, tous deux juristes et anciens directeurs de la KHRC, Makau wa Mutua, président de cette même organisation, ainsi que Paul Muite et Gibson Kamau Kuria, juristes (et pour le premier, homme politique), travaillant dans leurs cabinets d'avocats. Ces deux derniers ont des raisons à la fois militantes, mais aussi professionnelles et politiques, de s'intéresser à une affaire dont l'issue demeure très incertaine. Paul Muite et Gibson Kamau Kuria, engagés dans la préparation du procès au Kenya, sont deux juristes connus dans l'espace public kenyan pour leur engagement militant et politique depuis les années $1980^{38}$. Paul Muite, ancien protégé de Charles Njonjo, homme fort du régime kenyatta, est devenu un opposant radical au régime moi, par l'intermédiaire notamment de son accession à la présidence du barreau à la fin des années 1980. Député de l'opposition ensuite, proche des acteurs non étatiques de la contestation, il s'est aussi positionné comme leader kikuyu. Défenseur du nouveau régime Kibaki, il a donné l'impression depuis 2002 de revenir sur certaines des convictions qu'il défendait dans les années 1990, avant de nuancer son soutien au régime. Sa participation au procès peut être lue comme un moyen de consolider subtilement son image de défenseur et représentant du peuple kikuyu tout en jouant sur un militantisme moral et un nationalisme progressiste qui se manifeste par l'attaque de l'ancienne puissance coloniale. Gibson Kamau Kuria, qui est moins impliqué que Paul Muite dans cette action, ne s'est pas directement engagé en politique et a conservé l'image d'un juriste des droits de l'homme. Grand défenseur des prisonniers politiques des années 1980, lui-même emprisonné en 1987, il a continué à soutenir les actions des militants des droits de l'homme dans les années 1990, notamment autour de la révision

37. Stanley D. Ross, "The Rule of Law and Lawyers in Kenya", Journal of Modern African Studies, vol. 30, $\mathrm{n}^{\circ}$ 3, septembre 1992, p. 423-442.

38. Pour une analyse très critique des positionnements récents de ces deux juristes dans l'espace kenyan, voir G. R. Murunga et S. W. Nasong'o, "Bent on Self-Destruction... ", art. cité. Cet article les accuse d'être beaucoup moins vigilants sur les questions de justice depuis la mise en place de la nouvelle équipe gouvernementale qu'ils ont soutenue, et de mélanger allègrement les activités politiciennes, la défense d'hommes d'affaires, et de victimes de violations des droits de l'homme. 
constitutionnelle. Avocat, il est aussi un juriste d'affaires prospère qui a récemment défendu un homme d'affaires mis en cause dans une gigantesque affaire de corruption mêlant de nombreux personnages politiques kenyans. Sa participation, assez discrète, au procès mau mau peut être considérée comme un moyen de poursuivre ses activités militantes dans un contexte politique moins propice à l'activisme judiciaire et alors que ses activités de juriste d'affaires ont pris le pas sur sa réputation de défenseur des droits de l'homme. Un dernier avocat kenyan, Mbugua Mureithi, travaillant avec la KHRC depuis longtemps, s'occupe de la préparation technique de cette action judiciaire, en lien avec un membre de la KHRC. Les plaignants sont représentés en Grande-Bretagne par Martyn Day, du cabinet londonien Day, Leigh \& Co spécialisé, entre autres, dans la défense des droits de l'homme et notamment l'obtention de compensations pour des crimes passés. Après avoir gagné deux procès au nom d'anciens prisonniers britanniques au Japon et en Allemagne durant la Seconde Guerre mondiale, Martyn Day, s'est intéressé à des groupes kenyans pour lesquels il a plaidé à l'encontre de l'État britannique : en 2002 et 2003, il a obtenu des compensations financières pour des pasteurs samburu ayant été blessés par des mines laissées par l'armée britannique après des entraînements militaires. Il s'est également occupé d'une autre action en justice à propos de viols perpétrés par des militaires d'un camp britannique à l'encontre de femmes masaï et samburu. Cet activisme judiciaire international permet aux avocats, kenyans et britanniques, de combiner défense des droits de l'homme et actions juridiques, lucratives et professionnellement valorisantes.

Entre ces juristes et les vétérans mau mau, le lien a été noué par un personnage pivot de ces mobilisations, John Nottingham ${ }^{39}$. Britannique, ancien administrateur colonial au Kenya dans les années 1950, ayant donc assisté à l'insurrection et à la répression, il s'est attaché depuis les années 1960, de manière intermittente, à condamner la violence déployée par l'autorité qu'il avait servie. C'est lui qui, par exemple, a écrit l'un des premiers ouvrages sur l'insurrection mau mau, remettant en cause l'interprétation des Britanniques et qualifiant le soulèvement de combat nationaliste ${ }^{40}$. Installé

39. Entretien avec John Nottingham, Nairobi, 16 janvier 2007.

40. Carl Robserg et John Nottingham, The Myth of Mau Mau: Nationalism in Kenya, New York, Praeger, 1966. 
au Kenya, directeur d'une maison de publication, John Nottingham explique avoir saisi plusieurs opportunités pour tenter d'initier ce processus judiciaire: il a mis en contact Martyn Day, Caroline Elkins avec qui il échange beaucoup, la KHRC, qu'il pensait capable d'assurer le travail technique et militant autour de cette action judiciaire, et enfin des vétérans mau mau qui souhaitaient obtenir des compensations, et qu'il a aidé à s'organiser.

Les organisations et les avocats qui souhaitent intenter un procès à l'État britannique le font en effet au nom de groupes de vétérans mau mau, qui ont obtenu en 2003 l'autorisation légale de constituer des associations. Cette liberté d'action recouvrée était une condition importante de l'action légale. La question de leur unité a en revanche posé problème : avant de se regrouper au sein de la Mau Mau Veterans Association, les vétérans ont connu des concurrences acharnées, chacun revendiquant un rôle plus important dans la lutte, ou une indépendance politique plus marquée face aux anciens régimes ${ }^{41}$. Ces conflits proviennent des divisions du mouvement Mau Mau lui-même, et notamment de ces divisions territoriales. Certaines régions se sont investies davantage dans la lutte armée, d'autres ont réussi à capitaliser politiquement leur engagement après l'insurrection. Ces conflits ont été aggravés par les manipulations dont ces vétérans ont été l'objet de la part des Présidents Kenyatta et Moi, qui souhaitaient avoir la mainmise sur ces potentielles forces politiques, et ont longtemps affaibli la crédibilité de leurs revendications. Alors que les premières associations de vétérans fonctionnaient comme des coopératives foncières, elles ont ensuite été utilisées politiquement ${ }^{42}$. Le président Moi a voulu par exemple rallier certains segments de la population kikuyu en se prévalant du soutien de certaines associations, tout en les divisant. Il s'agissait aussi pour lui de contrer les discours dissidents, qui se revendiquaient des Mau Mau. Dans les années 1990, ces associations ont resurgi pour servir des luttes politiques en territoire kikuyu, et surtout en réaction aux conflits " ethniques ", politiques et fonciers qui ont vu les Kikuyus expulsés de leurs terres dans la province de la

41. "Wrangles Undermine the Demand for Payment by Mau Mau Veterans ", Daily Nation, 22 septembre 2003.

42. Josiah Mwangi Kariuki, "Mau Mau Associations in the 1990's ", L'Afrique orientale, annuaire 2003, Paris, L'Harmattan; Pau, CREPAO; Nairobi, IFRA, 2004, p. 375-396. 
Rift Valley ${ }^{43}$. L'alternance a permis de reconnaître légalement les associations de vétérans et la préparation du procès de les unir autour d'un objectif commun. La création de la Mau Mau Veterans Association est intervenue en 2003, après que certains vétérans eurent été victimes d'un personnage se présentant comme étant capable d'obtenir des compensations auprès de l'État britannique, et qui en avait profité pour leur extorquer de l'argent. Alors que John Nottingham cherchait à initier une action, en rencontrant Martyn Day et cet intermédiaire douteux, il tomba d'accord avec des vétérans mau mau pour mettre sur pieds une nouvelle association aujourd'hui dirigée par d'ex-combattants respectés : son président Ndungu wa Gicheru est connu pour avoir abattu un avion britannique durant l'insurrection et est reconnu comme l'un des guerriers mau mau importants dans nombre d'ouvrages historiques sur la question ; un autre leader de l'association, Gitu wa Kahengeri, a poursuivi, après l'indépendance, une carrière politique : il a été membre du Parlement de 1969 à 1974 et de 1979 à 1984, avant de rejoindre un parti d'opposition, le Ford People, au début des années 1990. Après les tentatives de malversation, la mise en place de cette association, qui s'appuie largement sur les organisations qui portent l'action judiciaire, confere aux vétérans une image redorée auprès du public, après les décennies de division et d'inaction. Les accusations et les conflits politiciens, ainsi que les enjeux financiers sont mis de côté pour laisser place à une association unique de vétérans dont l'objectif est de représenter les victimes et les héros de cette insurrection.

\section{Entre héroüsme et souffrances, l'efficacité mobilisatrice des victimes de violences politiques}

Si les Mau Mau sont devenus des symboles aisément manipulables dans l'espace public, tant par les gouvernants que par leurs opposants, c'est parce qu'ils font sens dans le langage politique commun aux citoyens kenyans. C'est en tout cas l'hypothèse énoncée par ceux qui réclament que les victimes oubliées de cet épisode soient érigées en héros nationaux. La figure de Dedan Kimathi, chef de guérilla et martyr, est devenue l'emblème de cette héroïsation contemporaine des Mau Mau. Dans un article consacré

43. C. Médard, "Les conflits "ethniques" au Kenya : une question de votes ou de terres? ", Afrique contemporaine, ${ }^{\circ} 180,1996$, p. 62-74. 
au renouveau du leadership politique, Willy Mutunga, juriste et militant des droits de l'homme, participe à cette lecture contemporaine du combat mau mau en distinguant, au sein de la société politique et civile actuelle, les " héros et héroïnes " d'un côté et les " traîtres ", apparentés aux loyalistes de l'époque de l'autre ${ }^{44}$. Cette distinction rejoint l'interprétation fournie par Caroline Elkins dans son entreprise d'identification historique des véritables victimes de cette insurrection, à qui le Kenya et la Grande Bretagne doivent reconnaissance. L'exclusivité héroïque conférée aux Mau Mau par ces initiatives ne fait pas l'unanimité, comme le rappelle l'ouvrage de David Anderson et d'autres avant lui. Par exemple, Bethwell Ogot, historien kenyan placé habituellement dans le camp des " conservateurs", propose une liste - non exhaustive - de personnages ayant participé, d'une manière ou d'une autre aux luttes contre le colonialisme ${ }^{45}$. Il mentionne des avocats, des syndicalistes, des hommes politiques et surtout des personnes provenant de groupes non kikuyu, afin de dénier aux seuls Mau Mau le droit de revendiquer un rôle central dans la lutte pour l'indépendance. Il affirme que c'est le refus de tenir compte de ces autres personnages importants de l'histoire politique qui tend à diviser la "mémoire collective " kenyane et rend difficile la constitution d'une mémoire nationale. David Anderson propose une solution de compromis : l'ensemble des squelettes de victimes de l'époque coloniale (Mau Mau et loyalistes, ou simples villageois) devraient être enterrés ensemble dans un Heroes Acre qui célébrerait la mémoire de toutes ces victimes de la violence coloniale. Sans expliquer l'équivalence faite entre victimes et héros, Anderson retire finalement toute substance à l'héroïsation qui, dans ce cas, ne fait pas de distinction entre les héros et les personnages ordinaires, voire les traitres. Sa démonstration empêche les usages politiques de l'histoire mau mau, et, si elle est saluée scientifiquement, risque de ne pas être aussi efficace

44. "S'agissant du passé des individus, nous soutenons que ceux qui étaient des "home guards" durant la guerre d'indépendance menée par les Mau Mau, et les autres Kenyans qui ont soutenu les forces britanniques contre les forces d'indépendance ne peuvent faire partie des leaders politiques alternatifs. Les hommes politiques qui ont trahi les intérêts kenyans de quelque manière que ce soit depuis l'indépendance appartiennent à cette même catégorie. [...] Les Kenyans aspirent à ce que ce soit leurs héroïnes et leurs héros qui les gouvernent, et non les traîtres, les opportunistes et les démolisseurs de toutes sortes ", in Kenya Human Rights Commission, Human Rights as Politics, KHRC, Nairobi, 2003.

45. Bethwell A. Ogot, "Mau Mau and Nationhood. The Untold Story ", in J. Lonsdale et A. E. S. Odhiambo (dir.), Mau Mau and Nationhood, op. cit., p. 8-36. 
politiquement que celle de Caroline Elkins. Car, ces mobilisations de victimes et ces débats sur les héros appartiennent au langage politique kenyan, qui alimente et renouvelle la critique politique. Cette dernière controverse historico-politique est en fait l'un des signes de cette vitalité de l'espace public ${ }^{46}$.

Marie-Émmanuelle Pommerolle est maître de conférences en science politique à l'Institut d'enseignement supérieur de Guyane (Université des Antilles et de la Guyane) et membre du Centre de recherche sur les pouvoirs locaux dans la Caraïbe (UMR 8053). Ses travaux portent sur l'action collective en contexte post-autoritaire et sur l'extraversion des mobilisations, notamment au Kenya et au Cameroun. Elle a publié dans de nombreuses revues (African Affairs, Africa Today, Politique africaine) et est membre du comité de rédaction de la revue Politique africaine.

\section{RÉSUMÉ}

Les mobilisations de victimes de violences coloniales : investigations historiques et judiciaires et débats politiques postcoloniaux au Kenya

Cette étude s'attache à décrire les processus de mobilisation autour d'ex-combattants mau mau insurgés contre la domination coloniale britannique au Kenya dans les années 1950. Cette résurgence du passé colonial dans les débats politiques contemporains, au Kenya comme dans son ancienne métropole, nous renseigne sur deux éléments spécifiques aux mobilisations lancées au nom de victimes revendiquées. D'abord, la qualification même de victime est sujette à controverse : ici, la recherche historique et l'action judiciaire sont mises à contribution pour affirmer le statut de victimes de ces vétérans mau mau, mais offrent des lectures contradictoires de cette victimisation. Ensuite, le statut de victime est l'objet d'usages multiples selon le lieu de son énonciation. Alors que des acteurs d'origines diverses (Kenyans, Britanniques, américains) participent à ces mobilisations et que ces dernières se déploient au Kenya comme en GrandeBretagne, les vétérans sont à la fois perçus comme des victimes d'une violence inouïe de la part de l'État colonial, et comme des héros dans le cadre national

46. Voir, sur la culture du débat politique au Kenya, même en période autoritaire, François Grignon, "La démocratisation au risque du débat ? Territoires de la critique et imaginaires politiques au Kenya (1990-1995)", in Denis-Constant Martin (dir.), Nouveaux langages du politique en Afrique Orientale, Paris, Karthala, 1998 ; Angelique Haugerud, The Culture of Politics in Modern Kenya, Cambridge, Cambridge University Press, 1995. 
kenyan où leurs mobilisations actuelles ré-affirment le combat pour l'indépendance, l'un des éléments du récit de l'identité nationale.

\section{The Mau Mau: Victims of colonial violence? Postcolonial historical and legal inquiries and political controversies in Kenya}

This study describes the current controversy over the role of the Mau Mau insurgents in the struggle against the British colonial domination of Kenya in the 1950s. The reemergence of the country's colonial past in present-day political debates, in Kenya as in Great Britain, points up two specific elements of the self-proclaimed victims' movement. First of all, the ascription of victimhood is controversial in and of itself: both historians and courts of law have been called on to assign the status of victims to these Mau Mau veterans, but they offer conflicting views on the validity of the claim. Secondly, the status of victim is used in several different ways according to the place of its proclamation. While parties of various origin (Kenyans, Britons, Americans) are involved in these movements both in Kenya and Great Britain, the veterans are perceived both as victims of unspeakable violence perpetrated by the colonial government and, at the same time, as heroes in the Kenyan national context, where their current mobilization reaffirms the struggle for independence, one of the key elements of the narrative of Kenya's national identity. 